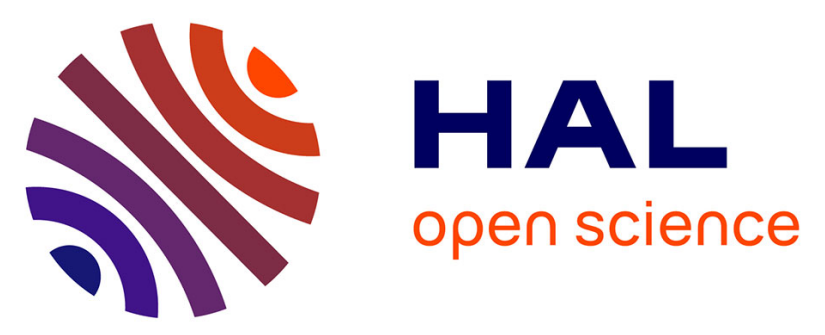

\title{
Viscoelastic effective properties for composites with rectangular cross-section fibers using the asymptotic homogenization method
}

Oscar Cruz-Gonzalez, Reinaldo Rodríguez-Ramos, José A. Otero, Julian Bravo-Castillero, Raúl Guinovart-Díaz, Raúl Martínez-Rosado, Federico J Sabina, Serge Dumont, Frédéric Lebon, Igor Sevostianov

\section{To cite this version:}

Oscar Cruz-Gonzalez, Reinaldo Rodríguez-Ramos, José A. Otero, Julian Bravo-Castillero, Raúl Guinovart-Díaz, et al.. Viscoelastic effective properties for composites with rectangular cross-section fibers using the asymptotic homogenization method. Generalized Models and Non-classical Approaches in Complex Materials 1, pp.203-222, 2018, 10.1007/978-3-319-72440-9_10 . hal-02336710

\author{
HAL Id: hal-02336710 \\ https://hal.science/hal-02336710
}

Submitted on 14 Jan 2020

HAL is a multi-disciplinary open access archive for the deposit and dissemination of scientific research documents, whether they are published or not. The documents may come from teaching and research institutions in France or abroad, or from public or private research centers.
L'archive ouverte pluridisciplinaire HAL, est destinée au dépôt et à la diffusion de documents scientifiques de niveau recherche, publiés ou non, émanant des établissements d'enseignement et de recherche français ou étrangers, des laboratoires publics ou privés. 


\title{
Viscoelastic effective properties for composites with rectangular cross-section fibers using the asymptotic homogenization method
}

\author{
Oscar L. Cruz-González, Reinaldo Rodríguez-Ramos, José A. Otero, Julián \\ Bravo-Castillero, Raúl Guinovart-Díaz, Raúl Martínez-Rosado, Federico J. Sabina, \\ Serge Dumont, Frederic Lebon, and Igor Sevostianov
}

\begin{abstract}
The present work deals with the estimation of the linear viscoelastic effective properties for composites with periodic structure and rectangular cross-section fibers, using the two-scale asymptotic homogenization method (AHM). As a particular case, the effective properties for a layered medium with transversely isotropic
\end{abstract}

\section{Oscar L. Cruz-González}

Facultad de Ciencias Técnicas, Departamento de Matemática, Universidad de Matanzas, Varadero road, Km. 2 1/2, Matanzas, Cuba

e-mail: oscar.lcg93@gmail.com

Reinaldo Rodríguez-Ramos · Julián Bravo-Castillero · Raúl Guinovart-Díaz

Facultad de Matemática y Computación, Universidad de La Habana, San Lázaro y L, Vedado, La Habana, CP 10400, Cuba

e-mail: reinaldo@matcom.uh.cu, jbravo@matcom.uh.cu, guino@matcom.uh.cu

José A. Otero · Raúl Martínez-Rosado

Tecnologico de Monterrey, Escuela de Ingenieria y Ciencias, Atizapan de Zaragoza, Estado de México, México,

e-mail: j.a.otero@itesm.mx, rrosado@itesm.mx

Federico J. Sabina

Instituto de Investigaciones en Matemáticas Aplicadas y en Sistemas, Universidad Nacional Autónoma de México, Apartado Postal 20-126, 01000 CDMX, México

e-mail: fjs@mym.iimas.unam.mx

Serge Dumont

Université de Nîmes, Institut de Mathématiques Alexander Grothendieck, CNRS, UMR 5149, CC.051, Pl. E. Bataillon, 34095 Montpellier Cedex 5, France

e-mail: serge.dumonteunimes. fr

Frederic Lebon

Aix-Marseille Univ., CNRS, Centrale Marseille, LMA, 4 Impasse Nikola Tesla, CS 40006, 13453 Marseille Cedex 13, France

e-mail: lebon@lma.cnrs-mrs.fr

Igor Sevostianov

Department of Mechanical and Aerospace Engineering, New Mexico State University, Las Cruces, NM 88003, USA

e-mail: igor@nmsu.edu 
properties are obtained. Two times the homogenization method, in different directions, according to the geometrical configuration of the composite material is applied for deriving the analytical expressions of the viscoelastic effective properties for a composite material with rectangular cross-section fibers, periodically distributed along one axis. In addition to that, models with different creep kernels, in particular, the Rabotnov's kernel are analyzed. Finally, the numerical computation of the effective viscoelastic properties is developed for the analysis of the results. Moreover, a numerical algorithm using FEM is developed in the present work. Comparisons with other approaches are given as a validation of the present model.

\subsection{Introduction}

Many materials like metals, polymers, and ceramics especially at high temperature exhibit time dependent behavior. For linear non ageing viscoelastic materials, the convolution product in the Stieltjes space is often used for modeling of the time dependent constitutive law. The overall behavior of viscoelastic composite materials is investigated by many researchers based on homogenization techniques. Classically, the investigation of effective properties of non ageing linear viscoelastic composites are mainly based on the correspondence principle and Laplace transform. This approach changes the convolution constitutive law describing the linear non ageing viscoelastic behavior into a fictitious linear elastic one in the Laplace domain. Linear homogenization method can then be used to drive the effective properties in the frequency domain. The time dependent effective properties can be obtained by performing numerical inversion of their Laplace transform. Based on this methodological approach, many researchers investigated the overall non ageing behavior of linear viscoelastic composites.

Important results obtained for elastic materials can be translated to linear non ageing viscoelastic materials using the correspondence principle (Hashin, 1965, 1970b; Christensen, 1969; Schapery, 1967; Wang and Weng, 1992; Kachanov, 1992; Lahellec and Suquet, 2007; Dormieux et al, 2006). It can be shown that in the Laplace-Carson (LC) transform space, the writing of the constitutive behavior is the same as in elasticity, the stiffness tensors being nevertheless functions of the LC transform variable (denoted thereafter LC variable). However, assuming that the solution in LC space is obtained, there are still considerable difficulties in obtaining the inverse LC transform to find the corresponding results in time space (Lévesque et al, 2007; Le et al, 2007).

Homogenization of viscoelastic composites can be performed analytically by solving the homogenization equations in Laplace-Carson space with the so-called correspondence principle (Hashin, 1966, 1970a; Laws and McLaughlin, 1978; Beurthey and Zaoui, 2000; Lévesque et al, 2007). The time domain solution is usually obtained with inversion algorithms,such as the collocation method (Schapery, 1964; Lévesque et al, 2007). The recently developed method of Lévesque et al (2007) is quite accurate, leads to thermodynamically admissible materials but requires a 
moderate computation time. Another approach consists of a time-integration approach, relying on variational principles (Lahellec and Suquet, 2007). While it avoids Laplace-Carson transforms and solves the viscoelastic problem directly in the time domain, its numerical implementation is challenging. Finally, another technique relies on a direct quasi-elastic approximation in the Laplace-Carson space (Brenner et al, 2002). This method is computationally-efficient but at the expense of accuracy. Finally, Ricaud and Masson (2009) have shown that the Laplace-Carson scheme, making use of the Prony series approximation, is equivalent to a time-integration scheme of the internal variable formulation, establishing a link between the two approaches.

This work is devoted to Gerard A. Maugin, who apported significant contributions in the micro-mechanic area. In particular, the authors had the pleasant opportunity to collaborate with him in the piezoelectric composites area (Berger et al, 2003, 2006; Otero et al, 2003). In the present contribution, making use of the two-scale asymptotic homogenization method (AHM) and correspondence principle, an equilibrium viscoelastic heterogeneous problem is solved similar to the elastic case, but in Laplace-Carson space. The overall behavior of composite non-ageing constituents is investigated with the estimation of the linear viscoelastic effective properties. As a particular case, the explicit formulae to predict the effective relaxation modulus for a two-layered medium with transversely isotropic properties are obtained. The numerical algorithm proposed by Hollenbeck (1998), to invert the Laplace transform, is using to calculate the properties to the homogenized composites in the time domain. Different creep kernels are analyzed, in particular, a time-dependent function considerated by Dischinger's model (see Maghous and Creus, 2003) and the fractionexponential function or Rabotnov's kernel (see Sevostianov et al, 2015, 2016) with the aim to make several comparison and to validate the numerical results. A model of finite element method is implemented and comparisons with the obtained results using finite element method is also realized. An alternative approach, using double homogenization scheme, is considered to estimate the linear viscoelastic effective properties for composites with periodic structure and rectangular cross-section fibers. This approach is inspired in the geometric design of the material.

\subsection{Statement of the Viscoelastic Heterogeneous Problem}

A heterogeneous material with periodic structure exhibiting a linear viscoelastic behavior is considered. It occupies a region $\Sigma$ in $\mathbb{R}^{3}$. Cartesian coordinate system $\boldsymbol{x}\left(x_{i}\right)$ is used where the stress tensor, external force field, surface force field, displacement field and outer unit normal to the boundary $\partial \Sigma$ of $\Sigma$ (see Fig. 10.1) are denoted by $\boldsymbol{\sigma}\left(\sigma_{i j}\right), \boldsymbol{f}\left(f_{i}\right), \boldsymbol{s}^{0}\left(s_{i}^{0}\right), \boldsymbol{u}\left(u_{i}\right)$ and $\boldsymbol{n}\left(n_{i}\right)$, respectively. The equilibrium equation under the action of external force field is written as (see Persson et al, 1993)

$$
\operatorname{div} \boldsymbol{\sigma}(\boldsymbol{x}, \mathrm{t})+\boldsymbol{f}(\boldsymbol{x})=\mathbf{0}, \quad \text { in } \quad \Sigma .
$$




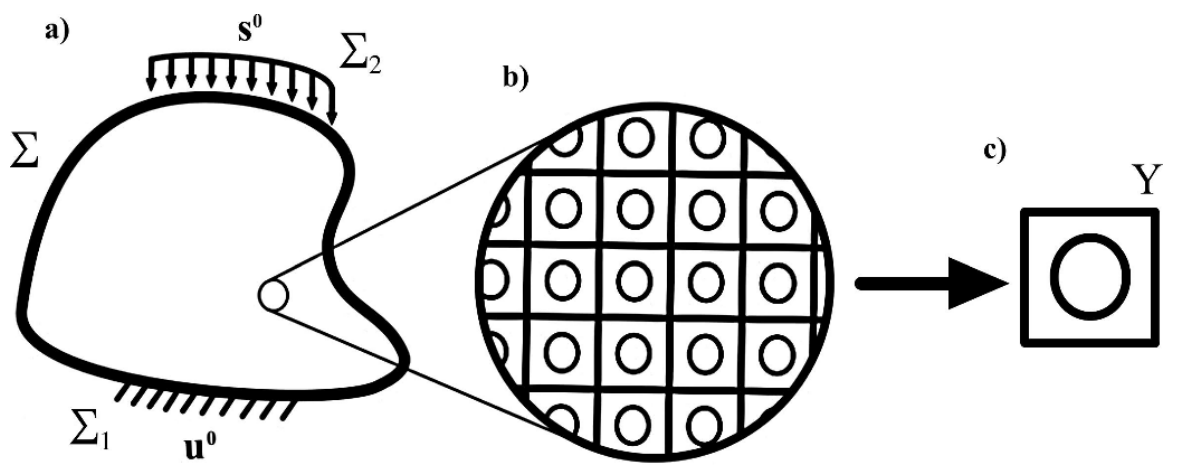

Fig. 10.1: Scales of the heterogeneous structure. a) Macroscopic heterogeneous structure, b) periodic microstructure and c) periodic cell.

The corresponding boundary conditions associated to (10.1) are

$$
\begin{array}{ccc}
\boldsymbol{u}(\boldsymbol{x}, t)=\boldsymbol{u}^{0}, & \text { on } & \Sigma_{1}, \\
\boldsymbol{\sigma}(\boldsymbol{x}, t) \cdot \boldsymbol{n}=\boldsymbol{s}^{0}, & \text { on } & \Sigma_{2} .
\end{array}
$$

The initial condition for Eq. (10.1) is

$$
\boldsymbol{u}(\boldsymbol{x}, t)=\mathbf{0}, \quad \text { in } \quad \Sigma \times\{0\}
$$

where $\Sigma_{1} \cup \Sigma_{2}=\partial \Sigma$ and $\Sigma_{1} \cap \Sigma_{2}=\varnothing$, (see Fig 10.1).

The stress and strain fields are linearly related to the constitutive law (see viscoelastic theory in Christensen, 1971; Pipkin, 1986)

$$
\boldsymbol{\sigma}(\boldsymbol{x}, t)=\int_{0}^{t} \boldsymbol{R}(\boldsymbol{x}, t-\tau): \frac{\partial \boldsymbol{\varepsilon}(\boldsymbol{u}(\boldsymbol{x}, \tau))}{\partial \tau} d \tau
$$

where $\boldsymbol{R}\left(R_{i j k l}\right)$ and $\boldsymbol{\varepsilon}\left(\varepsilon_{k l}\right)$ denote a fourth rank tensor (the creep kernel or relaxation modulus) and the Cauchy strain tensors, respectively.

The Eq. (10.5) can be expressed in a simplified form

$$
\boldsymbol{\sigma}(\boldsymbol{x}, t)=\boldsymbol{R}(\boldsymbol{x}, t) \circ \boldsymbol{\varepsilon}(\boldsymbol{u}(\boldsymbol{x}, t)),
$$

where $\circ$ is indicating the convolution integral (Zhang and Ostoja-Starzewski, 2015).

The following relationship is satisfied for small displacements

$$
\varepsilon_{k l}(\boldsymbol{u}(\boldsymbol{x}, t))=\frac{1}{2}\left(\frac{\partial u_{k}(\boldsymbol{x}, t)}{\partial x_{l}}+\frac{\partial u_{l}(\boldsymbol{x}, t)}{\partial x_{k}}\right) .
$$

Replacing (10.6) into (10.1) and using (10.2) - (10.4), the mathematical statement for the equilibrium viscoelastic heterogeneous problem is obtained 


$$
\begin{aligned}
& -\operatorname{div}(\boldsymbol{R}(\boldsymbol{x}, \mathrm{t}) \circ \boldsymbol{\varepsilon}(\boldsymbol{u}(\boldsymbol{x}, \mathrm{t})))=\boldsymbol{f}(\boldsymbol{x}), \\
& \boldsymbol{u}(\boldsymbol{x}, t)=\boldsymbol{u}^{0}, \quad \forall \boldsymbol{x} \in \Sigma_{1} \wedge \forall t \in \mathbb{R}, \\
& \boldsymbol{R}(\boldsymbol{x}, t) \circ \boldsymbol{\varepsilon}(\boldsymbol{u}(\boldsymbol{x}, t)) \cdot \boldsymbol{n}=\boldsymbol{s}^{0}, \quad \forall \boldsymbol{x} \in \Sigma_{2} \wedge \forall t \in \mathbb{R}, \\
& \boldsymbol{u}(\boldsymbol{x}, 0)=\mathbf{0}, \quad \forall \boldsymbol{x} \in \Sigma \text {. }
\end{aligned}
$$

The following additional conditions are considered:

1. $\boldsymbol{x}\left(x_{i}\right)$ is called the global coordinate. Also, is introduced the local or fast scale coordinate $\boldsymbol{y}\left(y_{i}\right)$, where $\boldsymbol{y}=\boldsymbol{\xi}^{-1} \boldsymbol{x}$. The parameter $\boldsymbol{\xi}$ is the fine mesh size of the cell structure (see Persson et al, 1993).

2. The relaxation modulus fulfills the memory principle $\boldsymbol{R}(\boldsymbol{x}, t) \rightarrow 0$ as $t \rightarrow \infty$ (see Sevostianov et al, 2016). In particular, we have $\boldsymbol{R}^{\xi}(\boldsymbol{x}, t)=\boldsymbol{R}(\boldsymbol{x} / \xi, t)=\boldsymbol{R}(\boldsymbol{y}, t)$ is $Y$-periodic related to the fast variable $y$.

3. $\boldsymbol{R}(\boldsymbol{y}, t) \in C^{\infty}\left(\mathbb{R}^{3} \times \mathbb{R}\right)$.

4. $\boldsymbol{\epsilon} \alpha, \beta, t_{0}$ such that $0 \leq \alpha \leq \boldsymbol{R}\left(\boldsymbol{y}, t_{0}\right) \leq \beta \leq \infty \forall \boldsymbol{y} \in \mathbb{R}^{3}(\xi \rightarrow 0)$.

5. $\boldsymbol{f}(\boldsymbol{x}) \in C^{\infty}(\Sigma)$.

The non-aging linear viscoelastic problem corresponds to elastic problems thanks to the Laplace-Carson transform (Lavergne et al, 2016). The transformed of a function $\boldsymbol{g}(\boldsymbol{x}, t)$ is defined by

$$
L_{C}[\boldsymbol{g}(\boldsymbol{x}, t)]=\widehat{\boldsymbol{g}}(\boldsymbol{x}, p)=p \int_{0}^{\infty} e^{-p t} \boldsymbol{g}(\boldsymbol{x}, t) d t .
$$

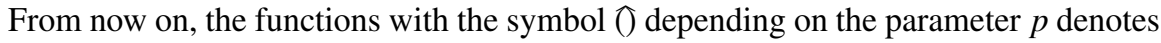
the Laplace-Carson space.

Considering the convolution theorem (see Sokolnikoff and Redheffer, 1968), the equilibrium viscoelastic heterogeneous problem (10.8)-(10.11) becomes,

$$
\begin{aligned}
P \widehat{\boldsymbol{u}}(\boldsymbol{x}, p) & =\boldsymbol{f}(\boldsymbol{x}), \\
\widehat{\boldsymbol{u}}(\boldsymbol{x}, p) & =\boldsymbol{u}^{0}, \quad \forall \boldsymbol{x} \in \Sigma_{1} \wedge \forall p \in[0, \infty], \\
\widehat{\boldsymbol{R}}(\boldsymbol{x}, p): \boldsymbol{\varepsilon}(\widehat{\boldsymbol{u}}(\boldsymbol{x}, p)) \cdot \boldsymbol{n} & =\boldsymbol{s}^{0}, \quad \forall \boldsymbol{x} \in \Sigma_{2} \wedge \forall p \in[0, \infty], \\
\widehat{\boldsymbol{u}}(\boldsymbol{x}, 0) & =\mathbf{0}, \quad \forall \boldsymbol{x} \in \Sigma,
\end{aligned}
$$

where

$$
P \widehat{\boldsymbol{u}}(\boldsymbol{x}, p)=-\operatorname{div}(\widehat{\boldsymbol{R}}(\boldsymbol{x}, \mathrm{p}): \boldsymbol{\varepsilon}(\widehat{\boldsymbol{u}}(\boldsymbol{x}, \mathrm{p})))
$$

\subsection{Two-Scale Asymptotic Homogenization Method to Solve the Heterogeneous Problem}

In this section the two-scales homogenization technique is used to obtain the basic equations and the effective characteristic of the composite. A formal asymptotic 
solution (see definition in Bakhvalov and Panasenko, 1989) for finding the solution of the problem is proposed. The asymptotic according to Bakhvalov and Panasenko (1989) for solving (10.12)-(10.15) is given in the form

$$
\widehat{\boldsymbol{u}}(\boldsymbol{x}, \xi, p)=\sum_{a=0}^{\infty} \xi^{a} \widehat{\boldsymbol{u}}^{(a)}(\boldsymbol{x}, \boldsymbol{y}, p),
$$

where $\widehat{\boldsymbol{u}}^{(a)}\left(\widehat{u}_{i}^{(a)}\right)$ is $Y$-periodic related to the variable $\boldsymbol{y} \forall a, \forall \boldsymbol{x} \in \Sigma, \forall p \in[0, \infty]$ and $\widehat{\boldsymbol{u}}^{(a)}(\boldsymbol{x}, \boldsymbol{y}, p) \in C^{\infty}\left(\Sigma \times \mathbb{R}^{3} \times[0, \infty]\right)$.

Besides, the expressions $e_{k l x}$ and $e_{k l y}$ are defined as follows (see Persson et al, 1993)

$$
\begin{aligned}
& \varepsilon_{k l x}(\widehat{\boldsymbol{\Phi}}(\boldsymbol{x}, p))=\frac{1}{2}\left(\frac{\partial \widehat{\Phi}_{k}(\boldsymbol{x}, p)}{\partial x_{l}}+\frac{\partial \widehat{\Phi}_{l}(\boldsymbol{x}, p)}{\partial x_{k}}\right) \\
& \varepsilon_{k l y}(\widehat{\boldsymbol{\Phi}}(\boldsymbol{y}, p))=\frac{1}{2}\left(\frac{\partial \widehat{\Phi}_{k}(\boldsymbol{y}, p)}{\partial y_{l}}+\frac{\partial \widehat{\Phi}_{l}(\boldsymbol{y}, p)}{\partial y_{k}}\right) .
\end{aligned}
$$

According to the chain rule and from (10.7), (10.16) - (1.18) can be obtained

$$
\varepsilon_{k l}\left(\widehat{\boldsymbol{u}}^{(a)}(\boldsymbol{x}, \boldsymbol{x} / \xi, p)\right)=\varepsilon_{k l x}\left(\widehat{\boldsymbol{u}}^{(a)}(\boldsymbol{x}, \boldsymbol{y}, p)\right)+\xi^{-1} \varepsilon_{k l y}\left(\widehat{\boldsymbol{u}}^{(a)}(\boldsymbol{x}, \boldsymbol{y}, p)\right) .
$$

Now, the objective is to find the expression of the coefficients such that the following equality is satisfied (Bakhvalov and Panasenko, 1989)

$$
P^{(\xi)} \widehat{\boldsymbol{u}}(\boldsymbol{x}, \xi, p)-\boldsymbol{f}(\boldsymbol{x})=\mathbf{O}(\xi) .
$$

In order to guarantee (10.20), the following operator is defined

$$
L_{\alpha \beta}(\bullet):=-\frac{\partial}{\partial \alpha_{j}}\left(\widehat{R}_{i j k l}(\boldsymbol{y}, p) \varepsilon_{k l \beta}(\bullet)\right)
$$

for $\alpha, \beta=\boldsymbol{x}, \boldsymbol{y}$ indistinctly and taken into account the rule for derivation

$$
\frac{\partial(\bullet)}{\partial x_{j}} \equiv \frac{\partial(\bullet)}{\partial x_{j}}+\frac{1}{\xi} \frac{\partial(\bullet)}{\partial y_{j}} .
$$

Then, replacing (10.16), (10.19) - (10.22) into (10.20), applying some simplifications and grouping in powers of $\epsilon$, the following sequence of problems are obtained

$$
\begin{aligned}
\xi^{-2} \rightarrow & L_{y y} \widehat{\boldsymbol{u}}^{(0)}(\boldsymbol{x}, \boldsymbol{y}, p)=\mathbf{0}, \\
\xi^{-1} \rightarrow & L_{x y} \widehat{\boldsymbol{u}}^{(0)}(\boldsymbol{x}, \boldsymbol{y}, p)+L_{y x} \widehat{\boldsymbol{u}}^{(0)}(\boldsymbol{x}, \boldsymbol{y}, p)+L_{y y} \widehat{\boldsymbol{u}}^{(1)}(\boldsymbol{x}, \boldsymbol{y}, p)=\mathbf{0}, \\
\xi^{0} \rightarrow & L_{x x} \widehat{\boldsymbol{u}}^{(0)}(\boldsymbol{x}, \boldsymbol{y}, p)+L_{x y} \widehat{\boldsymbol{u}}^{(1)}(\boldsymbol{x}, \boldsymbol{y}, p)+L_{y x} \widehat{\boldsymbol{u}}^{(1)}(\boldsymbol{x}, \boldsymbol{y}, p) \\
& +L_{y y} \widehat{\boldsymbol{u}}^{(2)}(\boldsymbol{x}, \boldsymbol{y}, p)-\boldsymbol{f}(\boldsymbol{x})=\mathbf{0} .
\end{aligned}
$$

The problems (10.23) - (10.25) can be solved in a recursive form. 
Considering the asymptotic (10.16) as an approximation of the exact solution of the original problem (10.12)-(10.15) and replacing (10.16) into the boundary conditions (10.13) and (10.14)

$$
\begin{gathered}
\widehat{\boldsymbol{u}}^{(0)}(\boldsymbol{x}, \boldsymbol{y}, p)=\boldsymbol{u}^{0}, \quad \forall \boldsymbol{x} \in \Sigma_{1} \wedge \forall p \in[0, \infty], \\
\widehat{\boldsymbol{R}}(\boldsymbol{x}, p): \boldsymbol{\varepsilon}\left(\widehat{\boldsymbol{u}}^{(0)}(\boldsymbol{x}, \boldsymbol{y}, p)\right) \cdot \boldsymbol{n}=\boldsymbol{s}^{0}, \quad \forall \boldsymbol{x} \in \Sigma_{2} \wedge \forall p \in[0, \infty] .
\end{gathered}
$$

Besides, the remaining terms are assigned by

$$
\begin{gathered}
\widehat{\boldsymbol{u}}^{(a)}(\boldsymbol{x}, \boldsymbol{y}, p)=\mathbf{0}, \quad \forall \boldsymbol{x} \in \Sigma_{1} \wedge \forall p \in[0, \infty] \wedge \forall a>0, \\
\widehat{\boldsymbol{R}}(\boldsymbol{x}, p): \boldsymbol{\varepsilon}\left(\widehat{\boldsymbol{u}}^{(a)}(\boldsymbol{x}, \boldsymbol{y}, p)\right) \cdot \boldsymbol{n}=\mathbf{0}, \quad \forall \boldsymbol{x} \in \Sigma_{2} \wedge \forall p \in[0, \infty] \wedge \forall a>0
\end{gathered}
$$

The conditions (10.26)-(10.29) are justified by construction because of the formal asymptotic solution is considered.

In order to solve (10.23)-(10.25) the following lemma is required. The proof of this lemma is given in Sect. 4.3 of Persson et al (1993).

Lemma 10.1. Let $\boldsymbol{F}\left(F_{i}\right)$ be square integrable function over $Y$ and consider the boundary value problem

$$
L_{y y} \boldsymbol{\Phi}=\boldsymbol{F},
$$

where $\boldsymbol{\Phi}$ is $Y$-periodic. Then the following conditions hold,

(i) A Y-periodic solution $\boldsymbol{\Phi}$ exists if and only if $\langle\boldsymbol{F}\rangle=0$.

(ii) If a $Y$-periodic solution $\boldsymbol{\Phi}$ exists, then it is unique up to a constant vector $\boldsymbol{c}$.

The notation $\langle\bullet\rangle$ defines the average over the $Y$-cell, i.e.,

$$
\langle\boldsymbol{F}\rangle:=\frac{1}{|Y|} \int_{Y} \boldsymbol{F} d y,
$$

where $|Y|$ is the measure of $Y$. Subsequently, the main results for each power of $\epsilon$ are summarized.

\subsubsection{Contribution of the Level $\xi^{-2}$ Problem}

The problem (10.23) and (10.26)-(10.27) is stated as follows

$$
\begin{array}{rlrl}
L_{y y} \widehat{\boldsymbol{u}}^{(0)}(\boldsymbol{x}, \boldsymbol{y}, p) & =\mathbf{0}, & & \\
\widehat{\boldsymbol{u}}^{(0)}(\boldsymbol{x}, \boldsymbol{y}, p) & =\boldsymbol{u}^{0}, & & \forall \boldsymbol{x} \in \Sigma_{1} \wedge \forall p \in[0, \infty], \\
\widehat{\boldsymbol{R}}(\boldsymbol{x}, p): \boldsymbol{\varepsilon}\left(\widehat{\boldsymbol{u}}^{(0)}(\boldsymbol{x}, \boldsymbol{y}, p)\right) \cdot \boldsymbol{n} & =\boldsymbol{s}^{0}, \quad & \forall \boldsymbol{x} \in \Sigma_{2} \wedge \forall p \in[0, \infty],
\end{array}
$$




$$
\widehat{\boldsymbol{u}}^{(0)}(\boldsymbol{x}, \boldsymbol{y}, 0)=\mathbf{0}, \quad \forall \boldsymbol{x} \in \Sigma
$$

Equation (10.30) has the trivial solution $\widehat{\boldsymbol{u}}^{(0)}(\boldsymbol{x}, \boldsymbol{y}, p) \equiv \mathbf{0}$. Thus, Lemma 10.1 indicates that $\widehat{\boldsymbol{u}}^{(0)}(\boldsymbol{x}, \boldsymbol{y}, p)$ is a solution of (10.30) if and only if it is a constant with respect to the variable $y$. It implies that,

$$
\widehat{\boldsymbol{u}}^{(0)}(\boldsymbol{x}, \boldsymbol{y}, p)=\widehat{\boldsymbol{v}}(\boldsymbol{x}, p)
$$

where $\widehat{\boldsymbol{v}}(\boldsymbol{x}, t)$ is a infinitely differentiable function (see Persson et al, 1993).

Now, from (10.31) - (10.33) are obtained

$$
\begin{array}{r}
\widehat{\boldsymbol{u}}^{(0)}(\boldsymbol{x}, \boldsymbol{y}, p)=\widehat{\boldsymbol{v}}(\boldsymbol{x}, p)=\boldsymbol{u}^{0}, \quad \forall \boldsymbol{x} \in \Sigma_{1} \wedge \forall p \in[0, \infty], \\
\widehat{\boldsymbol{R}}(\boldsymbol{x}, p): \boldsymbol{\varepsilon}(\widehat{\boldsymbol{v}}(\boldsymbol{x}, p)) \cdot \boldsymbol{n}=\boldsymbol{s}^{0}, \forall \boldsymbol{x} \in \Sigma_{2} \wedge \forall p \in[0, \infty], \\
\widehat{\boldsymbol{u}}^{(0)}(\boldsymbol{x}, \boldsymbol{y}, 0)=\widehat{\boldsymbol{v}}(\boldsymbol{x}, 0)=\mathbf{0}, \quad \forall \boldsymbol{x} \in \Sigma .
\end{array}
$$

\subsubsection{Contribution of the Level $\xi^{-1}$ Problem}

According to (10.34), the first term of (10.24) is zero, $L_{x y} \widehat{\boldsymbol{u}}^{(0)}(\boldsymbol{x}, \boldsymbol{y}, p)=L_{x y} \widehat{\boldsymbol{v}}(\boldsymbol{x}, p)=\mathbf{0}$. In this case, the problems (10.24) and (10.28)-(10.29) are reduced to

$$
\begin{aligned}
& L_{y y} \widehat{\boldsymbol{u}}^{(1)}(\boldsymbol{x}, \boldsymbol{y}, p)=-L_{y x} \widehat{\boldsymbol{u}}^{(0)}(\boldsymbol{x}, \boldsymbol{y}, p), \\
& \widehat{\boldsymbol{u}}^{(1)}(\boldsymbol{x}, \boldsymbol{y}, p)=\mathbf{0}, \quad \forall \boldsymbol{x} \in \Sigma_{1} \wedge \forall p \in[0, \infty], \\
& \widehat{\boldsymbol{R}}(\boldsymbol{x}, p): \boldsymbol{\varepsilon}\left(\widehat{\boldsymbol{u}}^{(1)}(\boldsymbol{x}, \boldsymbol{y}, p)\right) \cdot \boldsymbol{n}=\mathbf{0}, \quad \forall \boldsymbol{x} \in \Sigma_{2} \wedge \forall p \in[0, \infty], \\
& \widehat{\boldsymbol{u}}^{(1)}(\boldsymbol{x}, \boldsymbol{y}, 0)=\mathbf{0}, \quad \forall \boldsymbol{x} \in \Sigma \text {. }
\end{aligned}
$$

Applying the Lemma 10.1 on (10.38), having into account (10.34), the divergence theorem and the $Y$-periodicity condition of $\widehat{\boldsymbol{R}}(\boldsymbol{y}, p)$ (see the additional condition (2)), the following result is satisfied

$$
\left\langle-L_{y x} \widehat{\boldsymbol{u}}^{(0)}(\boldsymbol{x}, \boldsymbol{y}, p)\right\rangle=0 .
$$

Consequently, the existence of one solution for the problem (10.38) is guaranteed. Now, using separation of variables and the condition (ii) of Lemma 10.1, a general solution of (10.38) can be given by

$$
\widehat{\boldsymbol{u}}^{(1)}(\boldsymbol{x}, \boldsymbol{y}, p)=\widehat{\boldsymbol{N}}^{r s}(\boldsymbol{y}, p) \varepsilon_{r s x}(\widehat{\boldsymbol{v}}(\boldsymbol{x}, p))+\widehat{\boldsymbol{w}}(\boldsymbol{x}, p),
$$

where $\widehat{\boldsymbol{N}}^{r s}\left(\widehat{N}_{i}^{r s}\right)$ is called the local function and $\widehat{\boldsymbol{w}}\left(\widehat{w}_{i}\right)$ is infinitely differentiable function.

Finally, replacing (10.34) and (10.42) into (10.38) and after some simplifications the cell problem is obtained 


$$
-\frac{\partial}{\partial y_{j}}\left(\widehat{R}_{i j k l}(\boldsymbol{y}, p) \varepsilon_{k l y}\left(\widehat{\boldsymbol{N}}^{r s}(\boldsymbol{y}, p)\right)\right)=\frac{\partial}{\partial y_{j}}\left(\widehat{R}_{i j r s}(\boldsymbol{y}, p)\right),
$$

where, employing the Lemma 10.1 on (10.43), $\widehat{\boldsymbol{N}}^{r s}$ is $Y$-periodic function.

Having into account (10.35) - (10.37), (10.39) - (10.42), the boundary conditions and initial condition to the cell problem are written in term of the local function

$$
\begin{array}{r}
\widehat{\boldsymbol{N}}^{r s}(\boldsymbol{y}, p)=\mathbf{0}, \quad \forall \boldsymbol{y} \in Y \wedge \forall p \in[0, \infty], \\
\widehat{\boldsymbol{R}}(\boldsymbol{x}, p): \boldsymbol{\varepsilon}\left(\widehat{\boldsymbol{N}}^{r s}(\boldsymbol{y}, p)\right) \cdot \boldsymbol{n}=\mathbf{0}, \quad \forall \boldsymbol{y} \in Y \wedge \forall p \in[0, \infty], \\
\widehat{\boldsymbol{N}}^{r s}(\boldsymbol{y}, 0)=\mathbf{0}, \quad \forall \boldsymbol{y} \in \mathbb{R}^{3} .
\end{array}
$$

\subsubsection{Contribution of the Level $\xi^{0}$ Problem}

The problem (10.25) and (10.28)-(10.29) are given as follows

$$
\begin{gathered}
L_{y y} \widehat{\boldsymbol{u}}^{(2)}(\boldsymbol{x}, \boldsymbol{y}, p)=\boldsymbol{f}(\boldsymbol{x})-L_{x x} \widehat{\boldsymbol{u}}^{(0)}(\boldsymbol{x}, \boldsymbol{y}, p)-L_{x y} \widehat{\boldsymbol{u}}^{(1)}(\boldsymbol{x}, \boldsymbol{y}, p)-L_{y x} \widehat{\boldsymbol{u}}^{(1)}(\boldsymbol{x}, \boldsymbol{y}, p),\left(10, \quad \forall \boldsymbol{x} \in \Sigma_{1} \wedge \forall p \in[0, \infty]\right. \\
\widehat{\boldsymbol{u}}^{(2)}(\boldsymbol{x}, \boldsymbol{y}, p)=\mathbf{0}, \quad \forall \boldsymbol{\boldsymbol { x }} \in \Sigma_{2} \wedge \forall p \in[0, \infty], \\
\widehat{\boldsymbol{R}}(\boldsymbol{x}, p): \boldsymbol{\varepsilon}\left(\widehat{\boldsymbol{u}}^{(2)}(\boldsymbol{x}, \boldsymbol{y}, p)\right) \cdot \boldsymbol{n}=\mathbf{0}, \quad \forall \boldsymbol{x} \in \Sigma \\
\widehat{\boldsymbol{u}}^{(2)}(\boldsymbol{x}, \boldsymbol{y}, 0)=\mathbf{0}, \quad
\end{gathered}
$$

The Lemma 10.1 guarantees the existence of one $Y$-periodic solution of the problem (10.47), if and only if

$$
\left\langle\boldsymbol{f}(\boldsymbol{x})-L_{x x} \widehat{\boldsymbol{u}}^{(0)}(\boldsymbol{x}, \boldsymbol{y}, p)-L_{x y} \widehat{\boldsymbol{u}}^{(1)}(\boldsymbol{x}, \boldsymbol{y}, p)-L_{y x} \widehat{\boldsymbol{u}}^{(1)}(\boldsymbol{x}, \boldsymbol{y}, p)\right\rangle=0 .
$$

The functions $\widehat{\boldsymbol{R}}(\boldsymbol{y}, p)$ and $\widehat{\boldsymbol{N}}^{r s}(\boldsymbol{y}, p)$ are $Y$-periodic and the function $\widehat{\boldsymbol{v}}(\boldsymbol{x}, p)$ is independent of $\boldsymbol{y}$. Therefore, using (10.42) and the divergence theorem can be proved

$$
\left\langle L_{y x} \widehat{\boldsymbol{u}}^{(1)}(\boldsymbol{x}, \boldsymbol{y}, p)\right\rangle=0 .
$$

Then, from (10.51), the homogenized equation is obtained and it can be written in the form

$$
-\widehat{R}_{i j r s}^{(\mathrm{e})}(p) \frac{\partial}{\partial x_{j}} \varepsilon_{r s x}(\widehat{\boldsymbol{v}}(\boldsymbol{x}, p))=f_{i}(\boldsymbol{x})
$$

where

$$
\widehat{R}_{i j r s}^{(\mathrm{e})}(p)=\left\langle\widehat{R}_{i j r s}(\boldsymbol{y}, p)+\widehat{R}_{i j k l}(\boldsymbol{y}, p) \varepsilon_{k l y}\left(\widehat{\boldsymbol{N}}^{r s}(\boldsymbol{y}, p)\right)\right\rangle,
$$

are the homogenized coefficients or the effective coefficients. 
To obtain a well posed problem in (10.52), the boundary conditions and initial condition are needed for $\widehat{\boldsymbol{v}}(\boldsymbol{x}, p)$. From (10.35) and (10.36) the boundary conditions for the Eq. (10.52) are given in the form

$$
\begin{aligned}
\widehat{\boldsymbol{v}}(\boldsymbol{x}, p)=\boldsymbol{u}^{0}, & \forall \boldsymbol{x} \in \Sigma_{1} \wedge \forall p \in[0, \infty], \\
\widehat{\boldsymbol{R}}(\boldsymbol{x}, p): \boldsymbol{\varepsilon}(\widehat{\boldsymbol{v}}(\boldsymbol{x}, p)) \cdot \boldsymbol{n}=\boldsymbol{s}^{0}, & \forall \boldsymbol{x} \in \Sigma_{2} \wedge \forall p \in[0, \infty],
\end{aligned}
$$

and from (10.37) the initial condition is

$$
\widehat{\boldsymbol{v}}(\boldsymbol{x}, 0)=\mathbf{0}, \forall \boldsymbol{x} \in \Sigma .
$$

\subsection{Two Phase Viscoelastic Composite}

Now, we study a layered medium (see Fig 10.2). It is a composite formed by cells that are periodically distributed along one axis and each cell is made of a finite number of layers. In particular, we take $x_{3}$ the axis that describe the periodicity and it is perpendicular to the layers. The relaxation modulus $\widehat{\boldsymbol{R}}(\boldsymbol{x}, p)$ is periodic function of the coordinate $x_{3}$ and it does not depend on $x_{1}$ and $x_{2}$.

Expanding the cell problem (10.43) for the index $\mathrm{j}$, the first two terms are vanished because the fast variable depends only on $y_{3},\left(y_{3}=\xi^{-1} x_{3}\right)$, and therefore

$$
-\frac{\partial}{\partial y_{3}}\left(\widehat{R}_{i 3 k l}(\boldsymbol{y}, p) \varepsilon_{k l y}\left(\widehat{\boldsymbol{N}}^{r s}(\boldsymbol{y}, p)\right)+\widehat{R}_{i 3 r s}(\boldsymbol{y}, p)\right)=0 .
$$

The symmetry properties of the relaxation viscoelastic modulus are considered

$$
R_{i j k l}(\boldsymbol{x}, t)=R_{j i k l}(\boldsymbol{x}, t)=R_{i j l k}(\boldsymbol{x}, t)=R_{k l i j}(\boldsymbol{x}, t),
$$

where the last equality is consistent with the reciprocity principle stated by Onsager (see Maghous and Creus, 2003). Expanding (10.57) for the index $k$ and $l$, this equation

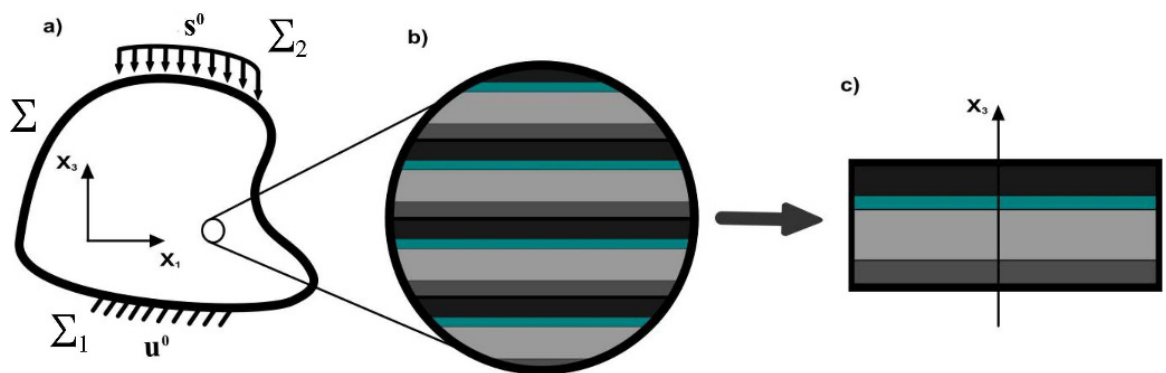

Fig. 10.2: Scales of a layered structure. a) Macroscopic structure, b) periodic microstructure, c) unit cell. 
becomes in the simplified form

$$
-\frac{\partial}{\partial y_{3}}\left(\widehat{R}_{i 3 k 3}(\boldsymbol{y}, p) \frac{\partial \widehat{N}_{k}^{r s}(\boldsymbol{y}, p)}{\partial y_{3}}+\widehat{R}_{i 3 r s}(\boldsymbol{y}, p)\right)=0 .
$$

From (10.58) is obtained

$$
\widehat{R}_{i 3 k 3}(\boldsymbol{y}, p) \frac{\partial \widehat{N}_{k}^{r s}(\boldsymbol{y}, p)}{\partial y_{3}}+\widehat{R}_{i 3 r s}(\boldsymbol{y}, p)=\widehat{A}_{i 3 r s}(p),
$$

and therefore,

$$
\frac{\partial \widehat{N}_{k}^{r s}(\boldsymbol{y}, p)}{\partial y_{3}}=\widehat{R}_{i 3 k 3}^{-1}(\boldsymbol{y}, p)\left(\widehat{A}_{i 3 r s}(p)-\widehat{R}_{i 3 r s}(\boldsymbol{y}, p)\right) .
$$

As $\widehat{\boldsymbol{N}}^{r s}$ is $Y$-periodic function, applying the average operator to both sides of (10.60) and using the divergence theorem we obtain

$$
\left\langle\frac{\partial \widehat{N}_{k}^{r s}(\boldsymbol{y}, p)}{\partial y_{3}}\right\rangle=0
$$

and consequently

$$
\widehat{A}_{i 3 r s}(p)=\left\langle\widehat{R}_{i 3 q 3}^{-1}(\boldsymbol{y}, p)\right\rangle^{-1}\left\langle\widehat{R}_{p 3 q 3}^{-1}(\boldsymbol{y}, p) \widehat{R}_{p 3 r s}(\boldsymbol{y}, p)\right\rangle .
$$

Replacing (10.62) into (10.60) and after some algebraic manipulations

$$
\begin{aligned}
\frac{\partial \widehat{N}_{k}^{r s}(\boldsymbol{y}, p)}{\partial y_{3}} & =\widehat{R}_{l 3 k 3}^{-1}(\boldsymbol{y}, p)\left\langle\widehat{R}_{l 3 q 3}^{-1}(\boldsymbol{y}, p)\right\rangle^{-1}\left\langle\widehat{R}_{p 3 q 3}^{-1}(\boldsymbol{y}, p) \widehat{R}_{p 3 r s}(\boldsymbol{y}, p)\right\rangle \\
& -\widehat{R}_{l 3 k 3}^{-1}(\boldsymbol{y}, p) \widehat{R}_{l 3 r s}(\boldsymbol{y}, p) .
\end{aligned}
$$

Using the formula of the effective coefficients (10.53)

$$
\begin{aligned}
\widehat{R}_{i j r s}^{(\mathrm{e})}(p) & =\left\langle\widehat{R}_{i j r s}(\boldsymbol{y}, p)+\widehat{R}_{i j k l}(\boldsymbol{y}, p) \varepsilon_{k l y}\left(\widehat{\boldsymbol{N}}^{r s}(\boldsymbol{y}, p)\right)\right\rangle \\
& =\left\langle\widehat{R}_{i j r s}(\boldsymbol{y}, p)+\widehat{R}_{i j k 3}(\boldsymbol{y}, p)\left(\frac{\partial \widehat{N}_{k}^{r s}(\boldsymbol{y}, p)}{\partial y_{3}}\right)\right\rangle .
\end{aligned}
$$

Replacing (10.63) into (10.64), the expression of the effective coefficients is given in the final form

$$
\begin{array}{r}
\widehat{R}_{i j r s}^{(\mathrm{e})}(p)=\left\langle\widehat{R}_{i j r s}(\boldsymbol{y}, p)\right\rangle+\left\langle\widehat{R}_{i j k 3}(\boldsymbol{y}, p) \widehat{R}_{l 3 k 3}^{-1}(\boldsymbol{y}, p)\right\rangle\left\langle\widehat{R}_{l 3 q 3}^{-1}(\boldsymbol{y}, p)\right\rangle^{-1} \\
\cdot\left\langle\widehat{R}_{p 3 q 3}^{-1}(\boldsymbol{y}, p) \widehat{R}_{p 3 r s}(\boldsymbol{y}, p)\right\rangle-\left\langle\widehat{R}_{i j k 3}(\boldsymbol{y}, p) \widehat{R}_{l 3 k 3}^{-1}(\boldsymbol{y}, p) \widehat{R}_{l 3 r s}(\boldsymbol{y}, p)\right\rangle .
\end{array}
$$


The Eq. (10.65) represents the general expression for the effective coefficients in Laplace-Carson space (see Pobedria, 1984).

In case of a two-layered medium where each constituent has isotropic characteristic, the expression of the effective coefficients derived from (10.57) are the following

$$
\begin{gathered}
\widehat{R}_{1111}^{(\mathrm{e})}(p)=\widehat{R}_{2222}^{(\mathrm{e})}(p) \\
=\langle\lambda+2 \mu\rangle+\left\langle\frac{1}{\lambda+2 \mu}\right\rangle^{-1}\left\langle\frac{\lambda}{\lambda+2 \mu}\right\rangle^{2}-\left\langle\frac{\lambda^{2}}{\lambda+2 \mu}\right\rangle, \\
\widehat{R}_{3333}^{(\mathrm{e})}(p)=\left\langle\frac{1}{\lambda+2 \mu}\right\rangle^{-1}, \\
\widehat{R}_{1122}^{(\mathrm{e})}(p)=\widehat{R}_{2211}^{(\mathrm{e})}(p)=\langle\lambda\rangle+\left\langle\frac{1}{\lambda+2 \mu}\right\rangle^{-1}\left\langle\frac{\lambda}{\lambda+2 \mu}\right\rangle^{2}-\left\langle\frac{\lambda^{2}}{\lambda+2 \mu}\right\rangle, \\
\widehat{R}_{1133}^{(\mathrm{e})}(p)=\widehat{R}_{3311}^{(\mathrm{e})}(p)=\widehat{R}_{3322}^{(\mathrm{e})}(p)=\widehat{R}_{2233}^{(\mathrm{e})}(p)=\left\langle\frac{\lambda}{\lambda+2 \mu}\right\rangle\left\langle\frac{1}{\lambda+2 \mu}\right\rangle^{-1}, \\
\widehat{R}_{1313}^{(\mathrm{e})}(p)=\widehat{R}_{2323}^{(\mathrm{e})}(p)=\left\langle\frac{1}{\mu}\right\rangle^{-1}, \\
\widehat{R}_{1212}^{(\mathrm{e})}(p)=\langle\mu\rangle .
\end{gathered}
$$

Notice that the global behavior is transversely isotropic for this type of laminate composite with isotropic constituents where their properties are denoted by the Lame's constants $\lambda$ and $\mu$.

\subsection{Numerical Results}

The above described method allows to find for two-layered viscoelastic medium with isotropic constituents, the value of the effective properties. In the literature, there are different creep kernels that describe the viscoelastic properties of a material. As examples, two different models are analyzed.

\subsubsection{Model I}

The first case is a two-layered medium (see Fig 10.3) structured by a layer 1 with linear elastic behavior and a layer 2 with viscoelastic behavior. This last layer is described by Dischinger's model (see Maghous and Creus, 2003). The Dischinger's model considers a time-dependent function, given in the form

$$
\varphi(\tau, t)=\exp (-\alpha t)-\exp (-\alpha \tau)
$$


The correspondent Lame's constants for the elastic layer 1 are taken by the relations

$$
\mu_{1}=\mu_{2,0}, \quad \lambda_{1}=K-\frac{2}{3} \mu_{2,0}
$$

and the relaxation functions for the viscoelastic layer 2 are defined for $\tau \leq t$ by

$$
\mu_{2}(\tau, t)=\mu_{2,0} \exp \left(\frac{\mu_{2,0}}{\alpha \beta} \varphi(\tau, t)\right), \quad \lambda_{2}(\tau, t)=K-\frac{2}{3} \mu_{2}(\tau, t),
$$

where $K$ is the bulk elastic modulus and $\mu_{2,0}$ represents a constant. Besides, $\alpha$ and $\beta$ are parameters of model. In Table 10.1 can be found the values of the parameters related to Dischinger's model. The results are obtained for $\tau=0$, a medium with non-ageing.

The computation of the effective coefficients are done using the formulae (10.66)(10.68) and (10.70). The behavior of the macroscopic properties are displayed in Fig. 10.4. This result by AHM is compared with Maghous and Creus (2003). Besides, in Fig. 10.4 are added the Voigt upper bound (VUB) and Reuss lower bound (RLB) to verify that the overall properties of the composite are between them. Since the composite has two phases and piecewise constant properties, the bounds can be calculated as follows

$$
\begin{aligned}
& \widehat{R}_{i j k l}^{(\text {Voigt })}(p)=\eta_{1} \widehat{R}_{i j k l}^{(1)}(p)+\eta_{2} \widehat{R}_{i j k l}^{(2)}(p), \\
& \widehat{R}_{i j k l}^{(\text {Reuss })}(p)=\left(\frac{\eta_{1}}{\widehat{R}_{i j k l}^{(1)}(p)}+\frac{\eta_{2}}{\widehat{R}_{i j k l}^{(2)}(p)}\right)^{-1},
\end{aligned}
$$

where $\eta_{1}$ and $\eta_{2}$ are the volume fractions of layer 1 and layer 2 respectively. The superscripts $(1),(2)$ in the kernels are indicating the corresponding material in each

Fig. 10.3 Cell of two-layered

\begin{tabular}{|c|c|c|c|c|c|}
\hline $\begin{array}{l}K \\
(\mathrm{GPa})\end{array}$ & $\begin{array}{l}\mu_{20} \\
(\mathrm{GPa})\end{array}$ & $\begin{array}{l}\alpha \cdot \beta \\
(\mathrm{GPa})\end{array}$ & $\begin{array}{l}\alpha \\
\left(\text { days }^{-1}\right)\end{array}$ & $\begin{array}{l}\eta_{1} \\
\text { (dimensionless) }\end{array}$ & $\begin{array}{l}\eta_{2} \\
\text { (dimensionless) }\end{array}$ \\
\hline 10000 & 8571 & 35667 & 0.026 & 0.5 & 0.5 \\
\hline
\end{tabular}
medium.

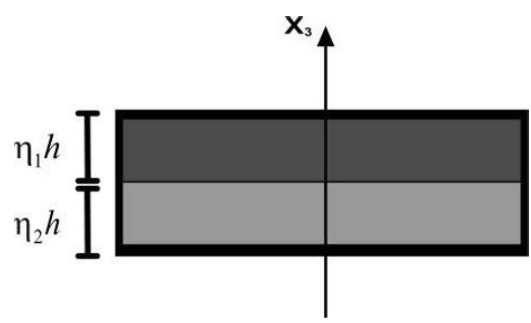

Table 10.1: Parameters of Dischinger's model. 

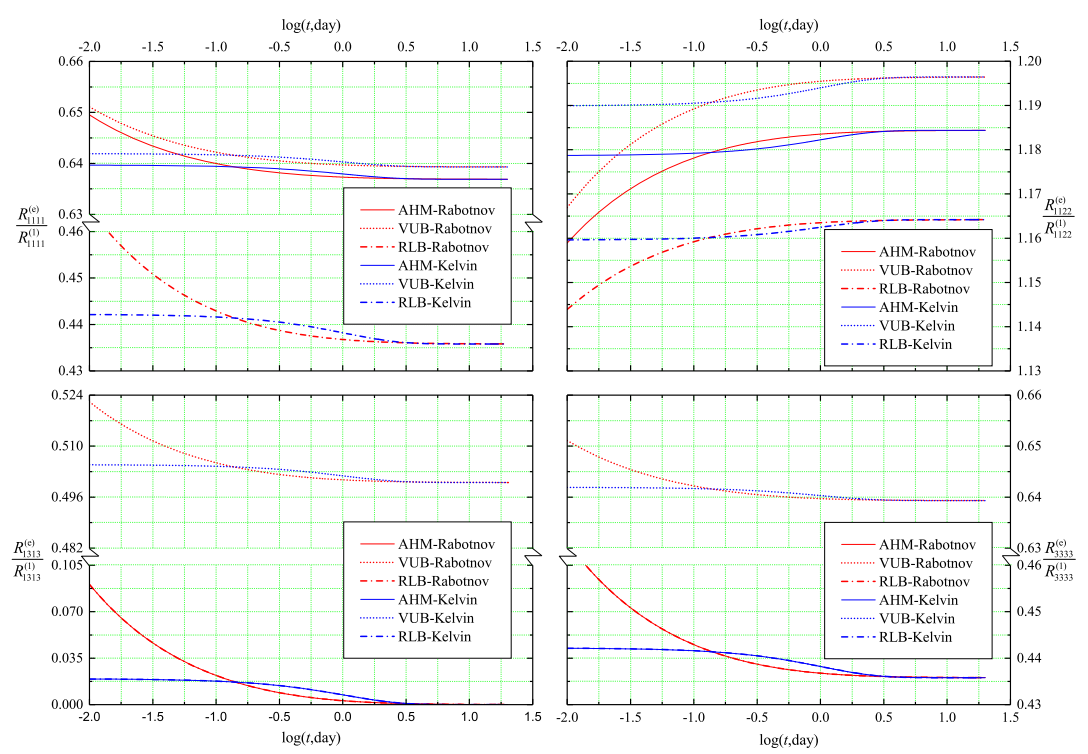

Fig. 10.4: Comparison between AHM, FEM, Maghous and Creus model and the Voigt and Reuss bounds. The results have been normalized with respect to the first phase and are obtained for $\tau=0$.

layer. In the computational process the numerical implementation of the effective coefficients is necessary. The function INVLAP2, developed in Matlab and the explanation given in Hollenbeck (1998), is adapted to guarantee the necessary technical support for the numerical inversion of Laplace-Carson transform.

As another validation of the present algorithm, a finite element method to compute homogenized coefficients, using Eqs. (10.58) and (10.61) written in the physical space, is implemented. For that purpose, the time interval $[0, T]$ is firstly discretized $t_{i}=k \Delta t,\left(k=0, \ldots, N_{T}\right)$, and the time integral is approximated using a midpoint quadrature rule. As a consequence, the kernel of the convolution is evaluated on a staggered grid. Then, we obtain a sequence of Poisson's equations that are discretized using a standard finite element method (for more details, see for example Chen et al, 2010; Dumont and Duval, 2013).

\subsubsection{Model II}

In the previous model, the proposed creep kernel is a composed exponential function. The main problem is that, simplest kernels as exponential function or their linear combinations, does not always describe correctly the viscoelastic behavior for de- 
termined materials (see Sevostianov et al, 2016). The use of fraction-exponential functions (see Rabotnov, 1948, 2014; Blair and Coppen, 1939, 1943)

$$
\ni_{\alpha}(\beta, t)=t^{-\alpha} \sum_{n=0}^{\infty} \frac{(-\beta)^{n} t^{n(1-\alpha)}}{\Gamma[(n+1)(1-\alpha)]},
$$

with $0 \leq \alpha<1$ and $0<\beta$, as kernel of viscoelastic operators, simplify and solve this situation. Furthermore, they allow, analytically, to obtain results with the use of Laplace transform and at the same time, offer an excellent concordance with the experimental data (see Sevostianov et al, 2016).

The algebraic properties of the Rabotnov's kernel are very well developed in Rabotnov (1977). One of the most important aspect described in the theory of this function is the analytical treatment of its Laplace transform

$$
L\left[\ni_{\alpha}(\beta, t)\right] \equiv \int_{0}^{\infty} \ni_{\alpha}(\beta, t) e^{-p t}=\frac{1}{p^{1-\alpha}+\beta} .
$$

Now, consider a two-layered medium with the structure: the layer 1 exhibits elastic properties and the layer 2 viscoelastic properties. The following data, obtained from Sevostianov et al (2016), has been selected to guarantee the numerical computation. The corresponding Lame's constants for the layer 1 are given as follows

$$
\mu_{1}=8.571 \mathrm{GPa}, \quad \lambda_{1}=K_{1}-\frac{2}{3} \mu_{1} \mathrm{GPa} .
$$

Taking the relaxation modulus of layer 2 as a fraction-exponential function or Rabotnov's kernel and applying the Laplace-Carson transform, having in mind the expression (10.71), the following analytic formulae are obtained,

$$
\widehat{\mu}_{2}(p)=p \cdot \mu_{20}\left(1+\frac{\gamma_{2}}{p^{\left(1-\alpha_{2}\right)}+\beta_{2}}\right), \quad \widehat{\lambda}_{2}(p)=K_{2}-\frac{2}{3} \widehat{\mu}_{2}(p),
$$

where $\mu_{20}$ is the instantaneous shear modulus and $\mu_{20}, \alpha_{2}, \beta_{2}$ and $\gamma_{2}$ are parameters of the material given experimentally. The material parameters are shown in Table 10.2.

The obtained effective coefficients (10.66)-(10.68) and (10.70), for this model, are displayed in Fig. 10.5. The expression (10.77) is reduced to exponential function for $\alpha=0$. In this case, it describes the properties of standard viscoelastic material or Kelvin model (see Sevostianov et al, 2015). Therefore, the numerical results of the effective coefficients by AHM are compared with the corresponding coefficients calculated by Kelvin's model (AHM-Kelvin). Besides, the Voigt upper bound (VUB) and Reuss lower bound (RLB) are added to the comparison (see Fig. 10.5) as a validation of the results obtained by the present model (AHM). 


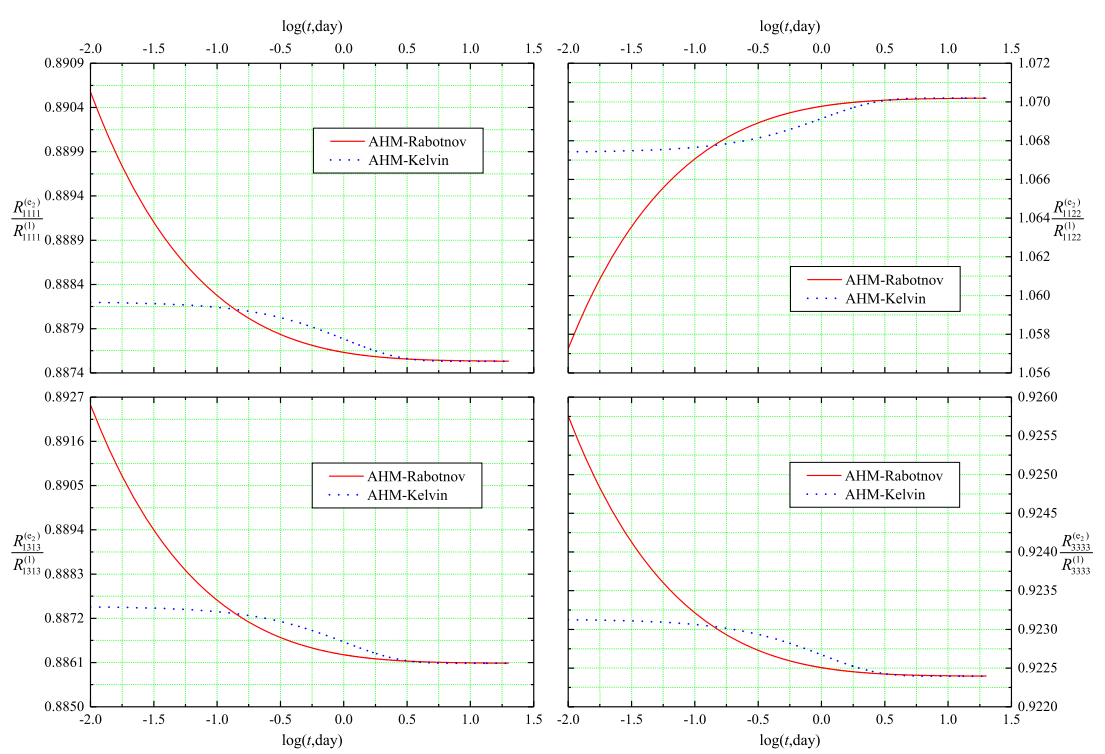

Fig. 10.5: Computation of the effective coefficients using Rabotnov's kernel. Comparison with Kelvin's model, Voigt and Reuss bounds. The coefficients have been normalized with respect to the first phase.

\subsubsection{Viscoelastic Effective Constants for Composites with Rectangular Cross-Section Fibers: Double Homogenization}

The purpose now is to obtain, using the asymptotic homogenization method, the viscoelastic effective properties for a composite material with rectangular cross-

Table 10.2: Material parameters.

\begin{tabular}{lllllll}
\hline Layer 1 & $\begin{array}{l}K_{1} \\
(\mathrm{GPa})\end{array}$ & $\begin{array}{l}\eta_{1} \\
(-)\end{array}$ & - & - & - & - \\
\hline & 10.0 & 0.5 & - & - & - & - \\
\hline Layer 2 & $K_{2}$ & $\eta_{2}$ & $\alpha_{2}$ & $\beta_{2}$ & $\gamma_{2}$ & $\mu_{20}$ \\
& $(\mathrm{GPa})$ & $(-)$ & $(-)$ & $\left(\right.$ day $\left.^{1-\alpha}\right)$ & $\left(\right.$ day $\left.^{1-\alpha}\right)$ & $(\mathrm{MPa})$ \\
\hline & 5.97 & 0.5 & 0.47 & 0.98 & 49.6 & 1.7
\end{tabular}




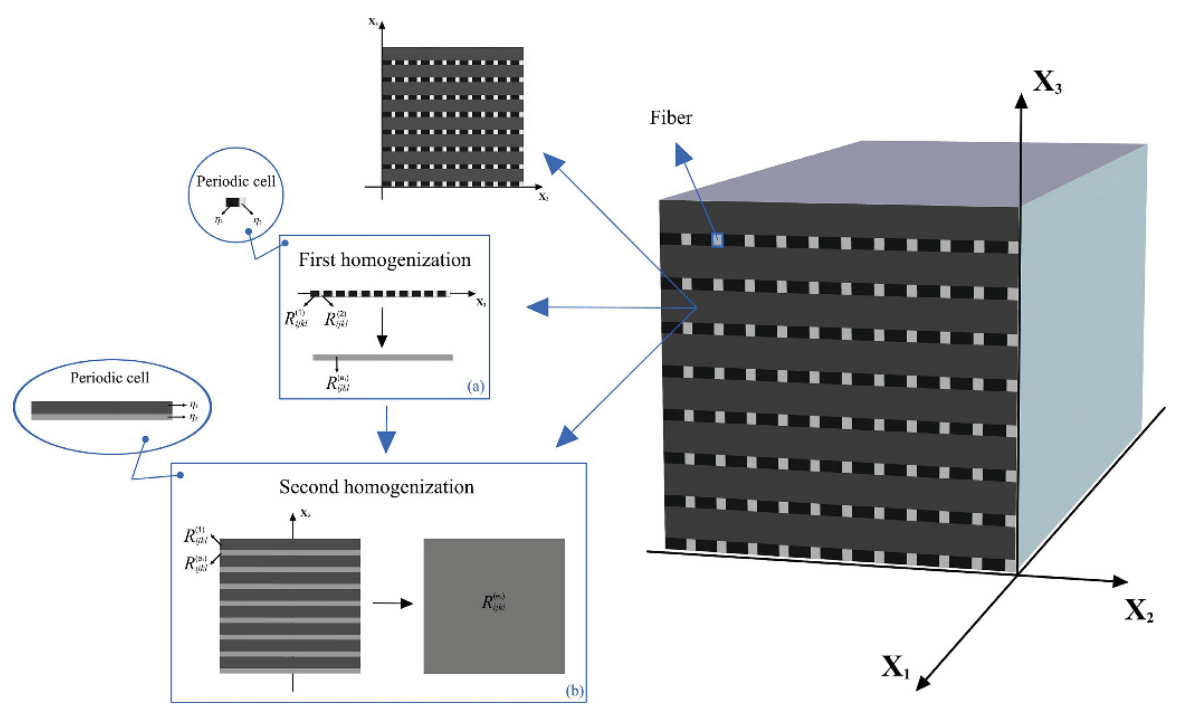

Fig. 10.6: Composites material with rectangular cross-section fibers. (a) First homogenization structure. (b) Second homogenization structure.

section fibers, periodically distributed (see Fig 10.6) along one axis with the aim of the aforementioned results applied to layered composites.

The basic idea is applied two times the homogenization method, in different directions, according to the geometrical configuration of the composite material. Therefore, we can divided the problem into two homogenization stages.

1. In a first moment, the composite is homogenized in the direction $x_{2}$. This structure can be analyzed as a two-layered medium with transversely isotropic properties after the homogenization process (see Fig. 10.6 (a)). In Sect. 10.4 is applied the homogenization method on this type of material in the direction $x_{3}$, obtaining the effective coefficients using (10.66)-(10.71). Now, as the problem is formulated in the direction $x_{2}$, it is affected the disposition of the subscripts in the formulae of the effective coefficients. In order to solve this issue, only it is necessary to change the subscripts $(3 \rightarrow 2)$ and $(2 \rightarrow 3)$ in all the formulae. Moreover, the superscript $\left(e_{1}\right)$ is added indicating the first homogenization step. For example, (10.71) is transformed as follows $\widehat{R}_{1313}^{\left(\mathrm{e}_{1}\right)}(p)=\langle\mu\rangle$. Clearly, for this two-layered medium $\langle f\rangle=\eta_{1} f^{(1)}+\eta_{2} f^{(2)}$, where the superscripts (1), (2) are indicating the corresponding layer (see Fig. 10.6 (a)).

2. Finally, the effective coefficients are calculated in the direction $x_{3}$. The structure can be analyzed as a two-layered medium (see Fig 10.6 (b)). In this case, the formulae obtained are just the same that (10.66)-(10.71). Besides, the notation $\left(\mathrm{e}_{2}\right)$ is added as superscript denoting the second homogenization step. For example, the coefficient $R_{1212}^{\left(\mathrm{e}_{2}\right)}(t)$ can be obtained from (10.71) as follows, $\widehat{R}_{1212}^{\left(\mathrm{e}_{2}\right)}(p)=\langle\mu\rangle$. Now, is necessary to take into account that $\langle f\rangle=\eta_{1} f^{(1)}+\eta_{2} f^{\left(\mathrm{e}_{1}\right)}$, where the 
superscript (1) indicates the elastic property of the layer 1 and the superscript $\left(e_{1}\right)$ denotes the effective viscoelastic property of the layer 2 . This last properties are calculated in the first homogenization step, see Fig. 10.6 (b).

In the Fig. 10.7 is displayed the computation of the effective coefficients for a composite material reinforced with rectangular cross-section fibers. A fractionexponential function (see Model II in Sect. 10.5) is assigned as relaxation modulus for the viscoelastic layer 2 . The volume fractions are taken as follow $\eta_{1}=0.7$ and $\eta_{2}=0.3$. The remaining data has been taken from Table 10.2.

\subsection{Conclusions}

In this work, the two-scale asymptotic homogenization method is applied to calculate the linear viscoelastic effective properties for layered composites where the distribution of the layers are perpendicular to one preferential axis. The local problems and the analytic expressions of the effective coefficients are derived. Based on this result, two times, the homogenization method is applied in different directions, according to the geometrical configuration of the composite material for deriving the analytical
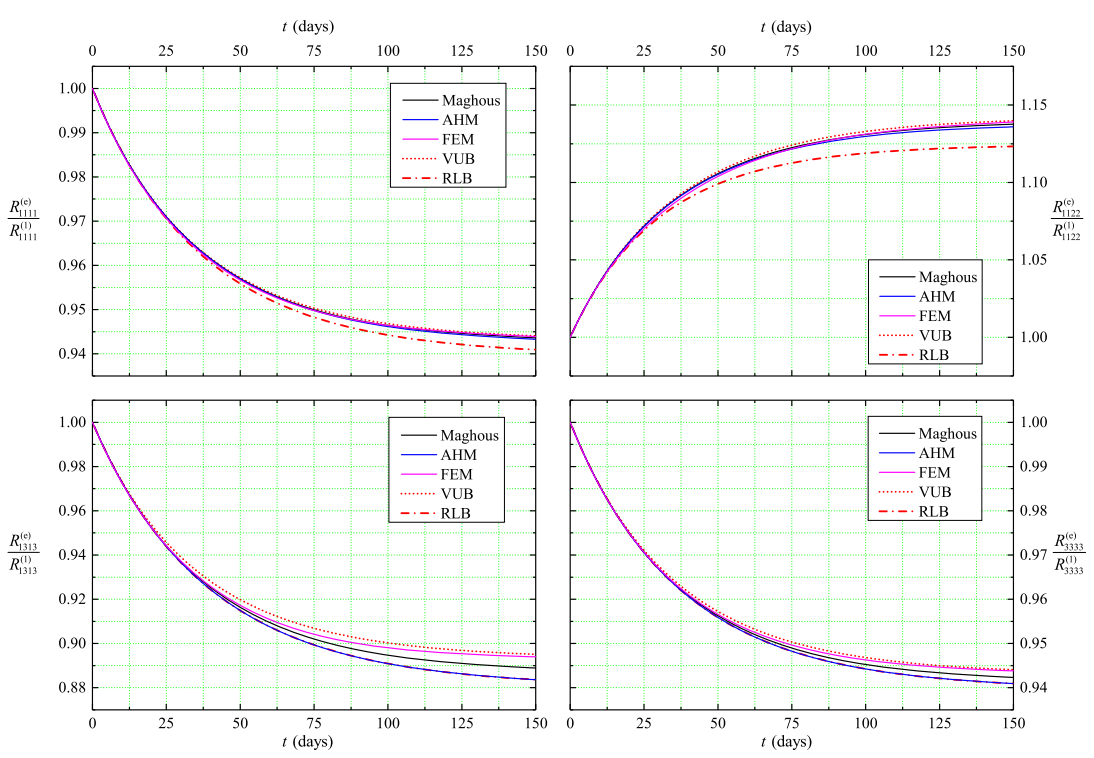

Fig. 10.7: Effective coefficients for rectangular cross-section fibers, using double homogenization, with Rabotnov's kernel. Comparison with the Kelvin's model. The results have been normalized with respect to the first phase. 
expressions of the viscoelastic effective properties for composites with rectangular cross-section fibers, periodically distributed along one axis. In addition, models with different creep kernels are studied, in particular, the Rabotnov's kernel is analyzed. A numerical computation where the inverse of Laplace-Carson is implemented numerically for the computation of the effective viscoelastic properties. An algorithm using FEM is developed in the present work and comparisons with other approaches are given as a validation of the present model.

Acknowledgements The authors would like to be grateful to University of Matanzas and Tecnologico de Monterrey, Escuela de Ingenieria y Ciencias, Atizapan de Zaragoza, Estado de Mexico, for its support. France-Cuba project "Partenariat Hubert Curien franco-cubain Carlos J. Finlay" 2017-2018 and Proyecto Nacional de Ciencias Básicas 2017-2019 are gratefully acknowledged. Thanks to Departamento de Matemáticas y Mecánica IIMAS-UNAM and FENOMEC for their support and Ramiro Chávez Tovar and Ana Pérez Arteaga for computational assistance.

\section{References}

Bakhvalov N, Panasenko GP (1989) Homogenisation: Averaging Processes in Periodic Media. Kluwer, Dordrecht

Berger H, Gabbert U, Köppe H, Rodriguez-Ramos R, Bravo-Castillero J, Guinovart-Diaz R, Otero JA, Maugin GA (2003) Finite element and asymptotic homogenization methods applied to smart composite materials. Computational Mechanics 33(1):61-67

Berger H, Kari S, Gabbert U, Rodriguez-Ramos R, Bravo-Castillero J, Guinovart-Diaz R, Sabina FJ, Maugin GA (2006) Unit cell models of piezoelectric fiber composites for numerical and analytical calculation of effective properties. Smart Materials and Structures 15(2):451-458

Beurthey S, Zaoui A (2000) Structural morphology and relaxation spectra of viscoelastic heterogeneous materials. European Journal of Mechanics - A/Solids 19(1):1-16

Blair GWS, Coppen FMV (1939) The subjective judgment of the elastic and plastic properties of soft bodies; the "differential thresholds" for viscosities and compression moduli. Proceedings of the Royal Society of London Series B, Biological Sciences 128(850):109-125

Blair GWS, Coppen FMV (1943) The estimation of firmness in soft materials. The American Journal of Psychology 56(2):234-246

Brenner R, Masson R, Castelnau O, Zaoui A (2002) A quasi-elastic affine formulation for the homogenised behaviour of nonlinear viscoelastic polycrystals and composites. European Journal of Mechanics - A/Solids 21(6):943-960

Chen M, Dumont S, Dupaigne L, Goubet O (2010) Decay of solutions to a water wave model with a nonlocal viscous dispersive term. Discrete and Continuous Dynamical Systems 27(4):1473-1492

Christensen RM (1969) Viscoelastic properties of heterogeneous media. Journal of the Mechanics and Physics of Solids 17(1):23-41

Christensen RM (1971) Theory of Viscoelasticity. Academic Press, New York

Dormieux L, Kondo D, Ulm FJ (2006) Microporomechanics. John Wiley \& Sons, Chichester

Dumont S, Duval JB (2013) Numerical investigation of asymptotical properties of solutions to models for waterways with non local viscosity. Int J Num Anal Modeling 10(2):333-349

Hashin Z (1965) Viscoelastic behavior of heterogeneous media. Trans ASME J Appl Mech 32:630636

Hashin Z (1966) Viscoelastic fibre reinforced materials. AIAA Journal 4:1411-1417

Hashin Z (1970a) Complex moduli of viscoelastic composites - I. General theory and application to particulate composites. Int J Solids Struct 6:539-552 
Hashin Z (1970b) Complex moduli of viscoelastic composites - II. Fibre reinforced materials. Int J Solids Struct 6:797-807

Hollenbeck KJ (1998) Invlap.m: a Matlab function for numerical inversion of Laplace transforms by the Hoog algorithm URL http: / / www . mathworks. com

Kachanov M (1992) Effective elastic properties of cracked solids: critical review of some basic concepts. Appl Mech Rev 45(8):304-335

Lahellec N, Suquet P (2007) Effective behavior of linear viscoelastic composites: A time-integration approach. International Journal of Solids and Structures 44(2):507 - 529

Lavergne F, Sab K, Sanahuja J, Bornert M, Toulemonde C (2016) Homogenization schemes for aging linear viscoelastic matrix-inclusion composite materials with elongated inclusions. International Journal of Solids and Structures 80:545 - 560

Laws N, McLaughlin R (1978) Self-consistent estimates for the viscoelastic creep compliances of composite materials. Proceedings of the Royal Society of London Series A, Mathematical and Physical Sciences 359(1697):251-273

Le QV, Meftah F, He QC, Le Pape Y (2007) Creep and relaxation functions of a heterogeneous viscoelastic porous medium using the Mori-Tanaka homogenization scheme and a discrete microscopic retardation spectrum. Mechanics of Time-Dependent Materials 11(3):309-331

Lévesque M, Gilchrist MD, Bouleau N, Derrien K, Baptiste D (2007) Numerical inversion of the Laplace-Carson transform applied to homogenization of randomly reinforced linear viscoelastic media. Computational Mechanics 40(4):771-789

Maghous S, Creus GJ (2003) Periodic homogenization in thermoviscoelasticity: case of multilayered media with ageing. International Journal of Solids and Structures 40(4):851 - 870

Otero JA, Bravo-Castillero J, Guinovart-Díaz R, Rodríguez-Ramos R, Maugin GA (2003) Analytical expressions of effective constants for a piezoelectric composite reinforced with square crosssection fibers. Arch Mech 55:357 - 371

Persson LE, Persson L, Svanstedt N, Wyller J (1993) The Homogenization Method. An Introduction. Student litteratur, Lund

Pipkin AC (1986) Lectures on Viscoelastic Theory. Springer, New York, Berlin, Heidelberg

Pobedria BE (1984) Mechanics of Composite Materials (in Russ.). Moscow State University Press, Moscow

Rabotnov YN (1948) Equilibrium of an elastic medium with after-effect (in Russ.). Prikladnaya Matematika i Mekhanika (J Appl Math Mech) 12(1):53 - 62

Rabotnov YN (1977) Elements of Hereditary Solid Mechanics. Mir, Moscow

Rabotnov YN (2014) Equilibrium of an elastic medium with after-effect. Fractional Calculus and Applied Analysis 17(3):684 - 696

Ricaud JM, Masson R (2009) Effective properties of linear viscoelastic heterogeneous media: Internal variables formulation and extension to ageing behaviours. International Journal of Solids and Structures 46(7):1599 - 1606

Schapery RA (1964) Application of thermodynamics to thermomechanical, fracture, and birefringent phenomena in viscoelastic media. Journal of Applied Physics 35(5):1451-1465

Schapery RA (1967) Stress analysis of viscoelastic composite materials. Journal of Composite Materials 1(3):228-267

Sevostianov I, Levin V, Radi E (2015) Effective properties of linear viscoelastic microcracked materials: Application of Maxwell homogenization scheme. Mechanics of Materials 84:28 - 43

Sevostianov I, Levin V, Radi E (2016) Effective viscoelastic properties of short-fiber reinforced composites. International Journal of Engineering Science 100:61 - 73

Sokolnikoff IS, Redheffer RM (1968) Mathematics of Physics and Modern Engineering. McGrawHill Book Company, Inc, New York, Toronto, London

Wang YM, Weng GJ (1992) The influence of inclusion shape on the overall viscoelastic behavior of composites. Trans ASME J Appl Mech 59(3):510 - 518

Zhang J, Ostoja-Starzewski M (2015) Mesoscale bounds in viscoelasticity of random composites. Mechanics Research Communications 68:98 - 104 